\title{
Dynamic Phases of Vortices in Superconductors with Periodic Pinning
}

\author{
C. Reichhardt, C. J. Olson, and Franco Nori \\ Department of Physics, The University of Michigan, Ann Arbor, Michigan 48109-1120
}

(May 10, 2018)

\begin{abstract}
We present results from extensive simulations of driven vortex lattices interacting with periodic arrays of pinning sites. Changing an applied driving force produces a rich variety of novel dynamical plastic flow phases which are very distinct from those observed in systems with random pinning arrays. Signatures of the transition between these different dynamical phases include sudden jumps in the current-voltage curves as well as marked changes in the vortex trajectories and vortex lattice order. Several dynamical phase diagrams are obtained as a function of commensurability, pinning strength, and spatial order of the pinning sites.
\end{abstract}

PACS numbers: 74.60.Ge, 74.60.Jg

The dynamics of driven vortex lattices interacting with quenched disorder have recently attracted considerable attention. Both theoretical [1] [5] and experimental [6] studies have suggested the exciting possibility that these systems exhibit novel dynamic phase transitions between different flow regimes as a function of driving force. In samples with random pinning, evidence for a transition from a plastic flow phase to a strongly driven ordered homogeneous phase has produced current debate over whether the strongly driven phase forms a moving crystal [2] or moving ordered glass [3, [4]. The dynamic phases may also be relevant to many other systems, such as Josephson junction arrays, charge-density waves, and electron crystals. Although much work has been done on dynamic phases in systems with random disorder, the case of periodic pinning has not been addressed. We show that driven vortex lattices interacting with periodic pinning exhibit a number of novel plastic flow phases which are not observed in random pinning arrays. Further, the onset of these different phases produces microscopic features in the vortex structure and flow patterns and gives rise to very pronounced features in macroscopically measurable current-voltage curves.

General interest in periodic arrays of pinning sites has increased now that it is possible to construct samples with well defined periodic pinning structures in which the microscopic pinning parameters, such as size, depth, periodicity, and density, can be carefully controlled $[9$. 9 . Interesting commensurability effects are observed both in magnetization measurements [7] and with direct imaging [8]. Periodic pinning arrays are also of technological importance since they can produce higher critical currents than an equal number of randomly placed pins 9].

When the pinning radius is much smaller than the lattice spacing, the "disorder" in the system can be fine tuned by changing the commensurability. At $B / B_{\phi}=1$, where $B_{\phi}$ is the field at which the number of vortices $N_{v}$ equals the number of pinning sites $N_{p}$, the vortex lattice locks into a periodic pinning array [7 9 and the pinning force is maximized. For $B / B_{\phi}>1$, the vortex lattice contains two species of vortices: the pinned vortices that are commensurate with the pinning array, and the generally more weakly pinned interstitial vortices that are caged by vortices at the pinning sites. When $B / B_{\phi}<1$, the vortex lattice contains a well defined number of $v a$ cancies which have their own dynamical behavior.

In order to study the two-dimensional (2D) dynamics of rigid flux lines driven over periodic arrays of pinning sites, we have performed a large number of $T=0$, current-driven molecular dynamics (MD) simulations. Unlike previous current-driven simulations [1.2, 2.,5], we examine the effects of periodic pinning arrays rather than random arrays, and cover a much larger range of the microscopic pinning and system parameters, allowing us to construct a series of detailed dynamical phase diagrams.

We numerically integrate the overdamped equations of motion [10]: $\mathbf{f}_{i}=\mathbf{f}_{i}^{v v}+\mathbf{f}_{i}^{v p}+\mathbf{f}_{d}=\eta \mathbf{v}_{i}$. Here $\mathbf{f}_{i}$ is the total force acting on vortex $i$ and we take $\eta=1$. The force from the other vortices is $\mathbf{f}_{i}^{v v}=\sum_{j=1}^{N_{v}} f_{0} K_{1}\left(\mid \mathbf{r}_{i}-\right.$ $\left.\mathbf{r}_{j} / / \lambda\right) \hat{\mathbf{r}}_{i j}$, where $K_{1}(r / \lambda)$ is a modified Bessel function, $\lambda$ is the penetration depth, and $\hat{\mathbf{r}}_{i j}=\left(\mathbf{r}_{i}-\mathbf{r}_{j}\right) /\left|\mathbf{r}_{i}-\mathbf{r}_{j}\right|$. A cut off is placed on $K_{1}(r / \lambda)$ after it reaches an extremely small value at $r=6 \lambda$. The pinning force is $\mathbf{f}_{i}^{v p}=\sum_{k=1}^{N_{p}}\left(f_{p} / r_{p}\right)\left|\mathbf{r}_{i}-\mathbf{r}_{k}^{(p)}\right| \Theta\left(\left(r_{p}-\left|\mathbf{r}_{i}-\mathbf{r}_{k}^{(p)}\right|\right) / \lambda\right) \hat{\mathbf{r}}_{i k}^{(p)}$. Here, $\Theta$ is the step function, $\mathbf{r}_{k}^{(p)}$ is the location of pinning site $k, f_{p}$ is the maximum pinning force, and $\hat{\mathbf{r}}_{i k}^{(p)}=$ $\left(\mathbf{r}_{i}-\mathbf{r}_{k}^{(p)}\right) /\left|\mathbf{r}_{i}-\mathbf{r}_{k}^{(p)}\right|$. The Lorentz force is modeled as a uniform force $\mathbf{f}_{d}$. All lengths, fields and forces are given in units of $\lambda, \Phi_{0} / \lambda^{2}$, and $f_{0}=\Phi_{0}^{2} / 8 \pi^{2} \lambda^{3}$, respectively.

We focus on experimentally accessible parameters that are close to those used in recent experiments [7]. Fixing the sample size at $36 \lambda \times 36 \lambda$, with periodic boundary conditions, and the pinning radius at $r_{p}=0.3 \lambda$, we examine an $18 \times 18$ square pinning array with $N_{p}=324$, giving a pinning density of $n_{p}=0.25 / \lambda^{2}$. We slowly increase the driving force $f_{d}$ along the horizontal symmetry axis ( $x$-axis) of the pinning lattice and compute the average velocity in the $x$-direction, $V_{x}=\left(1 / N_{v}\right) \sum_{i=1}^{N_{v}} \mathbf{v}_{i} \cdot \hat{\mathbf{x}}$. This quantity is proportional to a macroscopically measured voltage-current $V(I)$ curve. In order to separate the different effects that each of the pinning and system 
parameters have on the vortex dynamics, we fix all the parameters and vary only one at a time.

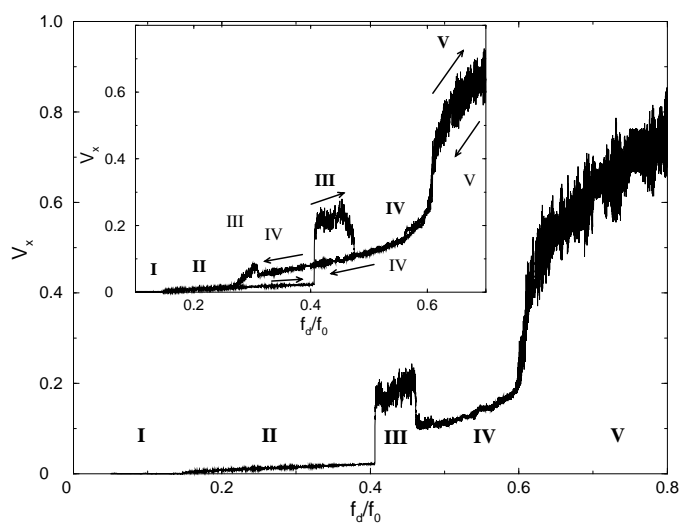

FIG. 1. Average vortex velocity $V_{x}$ versus driving force $f_{d}$, for $B / B_{\phi}=1.062, f_{p}=0.625 f_{0}, r_{p}=0.3 \lambda$, and $B_{\phi}=0.25 \Phi_{0} / \lambda^{2}$, with the pinning sites located in a square array; $f_{d}$ is increased from 0 to $0.8 f_{0}$. Several remarkable jumps in the curve can be clearly seen which correspond to transitions in the dynamical behavior of the driven lattice. To better identify the phases we have numbered them I-IV. The inset shows the hysteresis curve as $f_{d}$ is increased to $0.7 f_{0}$ and then decreased (phases now shown with unbold Roman numerals) to zero. Some phase boundaries, II-III and III-IV, show hysteresis while others do not.

In Fig. 1 we present a typical $V(I)$ curve for $B>B_{\phi}$ as the driving force is linearly increased from 0 to $0.8 f_{0}$ with $f_{p}=0.625 f_{0}, B_{\phi}=0.25 \Phi_{0} / \lambda^{2}$, and $B / B_{\phi}=1.062$. The $V(I)$ curve exhibits several remarkable features which clearly appear as discontinuous jumps and drops in $V_{x}$. We label each of the features as regions I through $\mathrm{V}$, and approximate the fraction of flux lines which are mobile at a specific driving force with: $\sigma=V_{x} / f_{d}$. As $f_{d}$ is increased in region I, $V_{x}$ is zero, indicating that the vortex lattice is pinned. At $f_{d}=0.146 f_{0}$, the onset of region II is marked by a finite $V_{x}$, caused by the depinning of interstitial vortices. Here, $\sigma=0.06$, confirming that only the interstitial vortices are mobile since the percentage of vortices above $B_{\phi}$ is also $\left(B-B_{\phi}\right) / B_{\phi}=0.06$. Region III begins at $f_{d}=0.406 f_{0}$, where a very sharp jump up in $V_{x}$ is seen, along with an increase in the number of mobile vortices to $\sigma=0.44$. The velocity fluctuations $\delta V_{x}$ are also much larger. At $f_{d}=0.462 f_{0}$, region IV appears with a sudden drop in $V_{x}$, with $\sigma=0.23$ and a reduction in $\delta V_{x}$. Finally, at $f_{d}=0.612 f_{0}$, just under the pinning force of each pinning site $\left(f_{p}=0.625 f_{0}\right)$, the entire lattice becomes mobile with $\sigma=1$, and the system enters region $\mathrm{V}$.

To further characterize the dynamic phases, we have performed a number of hysteresis runs where the driving force is slowly increased and then decreased. In the inset of Fig. 1 we show a typical curve for the system with the same parameters as in Fig. 1. There is little hysteresis for the transition IV-V. However, there is a very strong hysteresis at the transitions II-III and III-IV that persists in larger system sizes and also for low values of the spatial disorder. The hysteresis and the sharp jumps suggest that the II-III and III-IV phase boundaries might be first order. Details will be presented elsewhere [11].

We use a series of snapshots of the vortex position and vortex flow paths for regions II through $\mathrm{V}$ to show explicitly that the features in the $V(I)$ curve correspond to different plastic flow phases. Figure 2(a) shows the vortex trajectories in region II of the current-voltage curve from Fig. 1. It is clear that in region II only the interstitial vortices are mobile while the commensurate vortices remain pinned. The motion is confined to $1 D$ channels between the rows of pinning sites due to the square symmetry imposed by the pinned vortices. Such flow behavior for vortices in square pinning arrays has recently been experimentally observed [8].

Figure 2(b) illustrates that the vortex trajectories differ greatly from region III to II. The vortex lattice is now disordered, and the flow is no longer 1D but consists of channels that wind in both the $x$ and $y$ directions. Pin-topin motion also appears. Unlike the motion in region II, where only the interstitial vortices move and vortices at the pinning sites remain pinned, all the vortices in region III take part in the motion, with any one vortex moving for a time and then being temporarily trapped [12].

Another significant change in the vortex motion appears when the system enters region IV, as seen in Fig. 2(c). The vortex trajectories become more ordered and return to an exclusively $1 \mathrm{D}$ flow, with the mobile vortices moving along the pinning rows rather than between the rows as in region II. Only certain rows are mobile, and in these rows the additional vortices above $B_{\phi}$ leave their positions between the pinning rows to create moving incommensurate $1 D$ structures along the pinning row. An entire row does not move continuously, but instead a pulse appears in which only four vortices, near the incommensurate segment of the vortex row, are mobile. As this pulse or discommensuration moves across the sample, each vortex in the row is displaced by a single pinning lattice constant $a$. This disturbance is thus crossing the sample much more rapidly than the vortices themselves.

The vortex trajectories for region $\mathrm{V}$, in which the entire lattice is moving, are shown in Fig. 2(d). Some portions of the vortex lattice have a distorted triangular order, although the incommensurabilities from region IV are still present. The flow remains strictly 1D and along the pinning rows as in region IV, except that now all the rows are mobile. Rows with an incommensurate number of vortices move faster than the commensurate rows. As $f_{d}$ is increased further, the density of incommensurabilities and the vortex lattice structure do not change, so the system is always undergoing plastic flow and a moving crystal is not formed.

The onset of these different phases is described with force balance arguments that take into account the cou- 
pling of the two different species of vortices, interstitial and (pinned) commensurate. In region II, while the commensurate vortices remain pinned, the interstitial vortices begin flowing at a well defined driving force and exert a force $\mathbf{f}_{c-i c}$ on the pinned commensurate vortices. The total force on a commensurate vortex thus consists of the driving force $\mathbf{f}_{d}$, pinning force $\mathbf{f}_{p}$, and forces $\mathbf{f}_{c-c}$ from commensurate and $\mathbf{f}_{c-i c}$ interstitial vortices. The commensurate vortex will remain pinned as long the following inequality holds:

$$
\left|\mathbf{f}_{p}\right|>\left|\mathbf{f}_{d}+\mathbf{f}_{c-i c}+\mathbf{f}_{c-c}\right| .
$$

Since here we are using a square pinning lattice, from symmetry we have $\mathbf{f}_{c-c}=0$. If there are no incommensurate vortices, $\mathbf{f}_{c-i c}=0$, and commensurate vortices depin at $\left|\mathbf{f}_{d}\right|=\left|\mathbf{f}_{p}\right|$. When interstitial vortices are present, the term $\mathbf{f}_{c-i c}$ causes commensurate vortices to depin before $\left|\mathbf{f}_{d}\right|=\left|\mathbf{f}_{p}\right|$. These vortices depin more vortices so that the number of mobile vortices increases and region III appears. As long as $\left|\mathbf{f}_{d}\right|<\left|\mathbf{f}_{p}\right|$, not all the vortices will be mobile so that $\sigma<1$. For the parameters used in Fig. 1, the density of interstitial vortices is sufficiently low that they do not interact significantly with each other. In this case, we can solve Eq. (1) for the transition from region II to region III to give

$$
f_{p}=\left[f_{d}^{2}+f_{0}^{2} K_{1}^{2}\left(\frac{a+r_{p}}{2 \lambda}\right)\right]^{1 / 2} .
$$

With the parameters for the onset of region III, Eq. (2) gives $f_{p}=0.621 f_{0}$, which is in very good agreement with the value of $f_{p}=0.625 f_{0}$ used in the simulation.

The appearance of $1 \mathrm{D}$ motion exactly along the rows of pins in region IV might seem counterintuitive since for $B / B_{\phi}>1$ and $f_{d}=0$, when the vortices are not moving, an incommensurate vortex located along a pinning row is unstable to perturbations in the $y$-direction and will fall into the interstitial area between rows. For moving vortices, the situation is quite different since the vortices spend part of their time in the pinning sites. The pinning sites create a stabilizing force against perturbations in the transverse direction, confining the motion along the pinning rows. When the density of interstitial vortices is low, the onset of region IV occurs when the driving force is strong enough that interstitial vortices can depin commensurate vortices from a distance $a / 2$ in the longitudinal direction. This distance, and especially the repulsion from the remaining pinned vortices, allow the interstitial vortex to move towards the just-vacated pin site. For sufficiently strong $f_{d}$, it will remain moving along the pinned rows [11. The transition from region III to region IV should occur at

$$
f_{p}=\left[\left(f_{d}+\frac{f_{0}}{\sqrt{2}} K_{1}\left(r_{1}\right)\right)^{2}+\frac{f_{0}^{2}}{2} K_{1}^{2}\left(r_{1}\right)\right]^{1 / 2},
$$

where $r_{1}=\left(a / \sqrt{2}+r_{p}\right) / \lambda$. With the parameters used in Fig. 1 at the onset of the 1D incommensurate flow, Eq. (3) gives $f_{p}=0.624 f_{0}$, which is in very good agreement with the numerical value for $f_{p}$ shown in Fig. 1 .

To better characterize the flow behavior, we systematically vary $f_{p}$ with the rest of the parameters fixed. The resulting phase diagram in Fig. 3(a) outlines the onset of the different dynamical phases. As $f_{p}$ is increased, region I saturates at a value of $f_{d} \approx 0.146 f_{0}$. This occurs because, although the pinning force $f_{p}$ of the pinning sites is being increased, the vortex-vortex interactions which determine the interstitial pinning force are not changed. Region II only occurs when $f_{p}>0.37 f_{0}$, since for $f_{p}<0.37 f_{0}$, Eq. (2) cannot be satisfied even for very low $f_{d}$, and as soon as the interstitial vortices move they start to depin commensurate vortices. The same argument applies for the onset of region IV, which extends to even lower $f_{p}$ values. The II-III phase boundary follows Eq. (2), which for high $f_{d}$ goes as $f_{p} \approx f_{d}$, in agreement with the phase diagram. Similarly, the III-IV boundary follows Eq. (3), which is also linear for large $f_{d}$. The onset of region $\mathrm{V}$ also goes as $f_{p} \propto f_{d}$.

Next we vary the commensurability, from $B / B_{\phi}=0.75$ to 1.7 , producing the phase diagram in Fig. $3(\mathrm{~b})$. Just above $B / B_{\phi}=1$, the five phases of Fig. 1 are present. As $B / B_{\phi}$ is increased, the disordered-flow region III grows while the ordered-flow regions II and IV shrink. This is expected since an increase in $B / B_{\phi}$ effectively introduces more disorder via the addition of more interstitial vortices. For $B / B_{\phi}>1.3$, the flow becomes more disordered, the II-III phase boundary becomes ill-defined, and the flow in region $\mathrm{V}$ is no longer composed of $1 \mathrm{D}$ incommensurate flow along the pinning rows but has a number of vortices flowing between the pinning rows. At $B / B_{\phi}=1$, the commensurate case, we find only two phases: pinned and flowing, with the onset of flow occurring at $f_{d} \approx f_{p}$. The vortex flow for the commensurate case is elastic since, unlike the case for $B / B_{\phi}>1$, there are no discommensurations that cause certain rows to move faster. For $B / B_{\phi}<1$, where a number of vacancies appear in the vortex lattice, we observe a new vacancy flow phase, marked region VI. The depining force for the onset of vacancy motion is considerably higher than that for the onset of interstitial motion, in agreement with experiments [8]. Flow in $\mathrm{V}^{\prime}$ is like in $\mathrm{V}$, but now the faster-moving rows have vacancies.

Figure 3(c) shows a phase diagram in which the positions of the pinning sites are gradually disordered by randomly displacing them up to an amount $\delta r$ from the ordered pinning lattice position. In terms of the pinning lattice constant $a$, we consider the case $\delta r=a / 2$ to be a good approximation to a random pinning array. The disordered region III grows and dominates the phase diagram for disorder greater then $\delta r=a / 6$, so that only three phases occur, in agreement with other simulations of random pinning arrays [2, 4. 5 . 
Figure $3(\mathrm{~d})$ shows the phase diagram for increasing both vortex $n_{v}$ and pin $n_{p}$ density, determined by $B_{\phi}$. Here, the ordered-flow phase IV grows while the disordered flow region III shrinks. Increasing $B_{\phi}$, increases $n_{v}$ and thus effectively decreases $f_{p}$. As seen in Fig. 3(a), this suppresses II and III, while favoring IV. Phase diagrams for varying pinning radius $r_{p}$, angle of drive, temperature, and triangular pinning arrays will be presented and discussed in detail elsewhere 11].

In conclusion, we have demonstrated several novel dynamical phases which are very distinct from those found in random pinning arrays. These phases are marked by pronounced changes in the $V(I)$ curves and noise fluctuations which should be very accessible experimentally. We have directly related these features to the pronounced changes in the vortex lattice structure and flow pattern. These phases result from the coupling between two species of vortices. We have shown that certain phases show strong hysteresis while others do not. The dependence of these phases on various pinning and system parameters has been extensively studied and summarized in a series of phase diagrams [11]. We hope that these results encourage the experimental search of these new dynamic phases.

Computing services were provided by the University of Michigan Center for Parallel Computing, partially funded by NSF grant CDA-92-14296.

[1] H.J. Jensen, Y. Brechet, and A. Brass, J. Low Temp. Phys. 74, 293 (1989); A.-C. Shi and A.J. Berlinsky, Phys. Rev. Lett. 67, 1926 (1991).

[2] A.E. Koshelev and V.M. Vinokur, Phys. Rev. Lett. 73, 3580 (1994).

[3] T. Giamarchi and P. Le Doussal, Phys. Rev. Lett. 76, 3408 (1996).

[4] K. Moon, R.T. Scalettar and G.T. Zimányi, Phys. Rev. Lett. 77, 2778 (1996); S. Ryu et al., ibid 5115 (1996).

[5] N. Gronbech-Jensen, A.R. Bishop, and D. Dominguez, Phys. Rev. Lett. 76, 2985 (1996); M.C. Faleski, M.C. Marchetti and A.A. Middleton, Phys. Rev. B 54, 12427 (1996).

[6] U. Yaron et al., Nature 376, 753 (1995); A.C. Marley, M.J. Higgins and S. Bhattacharya, Phys. Rev. Lett. 74, 3029 (1995); M.C. Hellerqvist et al., ibid. 76, 4022 (1996).

[7] M. Baert et al., Phys. Rev. Lett. 74, 3269 (1995); E. Rosseel et al., Phys. Rev. B 53, R2983 (1996); V.V. Moshchalkov et al., ibid 7385 (1996); J.Y. Lin et al., ibid, R12714 (1996).

[8] K. Harada et al., Science 274, 1167 (1996).

[9] C. Reichhardt, et al., Phys. Rev. B 53, R8898 (1996); ibid, 54, 16108 (1996).

[10] G. Blatter, et al., Rev. Mod. Phys. 66, 1125 (1994).

[11] C. Reichhardt, C.J. Olson, F. Nori, unpublished. In [11].

[12] Videos illustrating the different dynamical phases described here are available at:

http://www-personal.engin.umich.edu/ nor
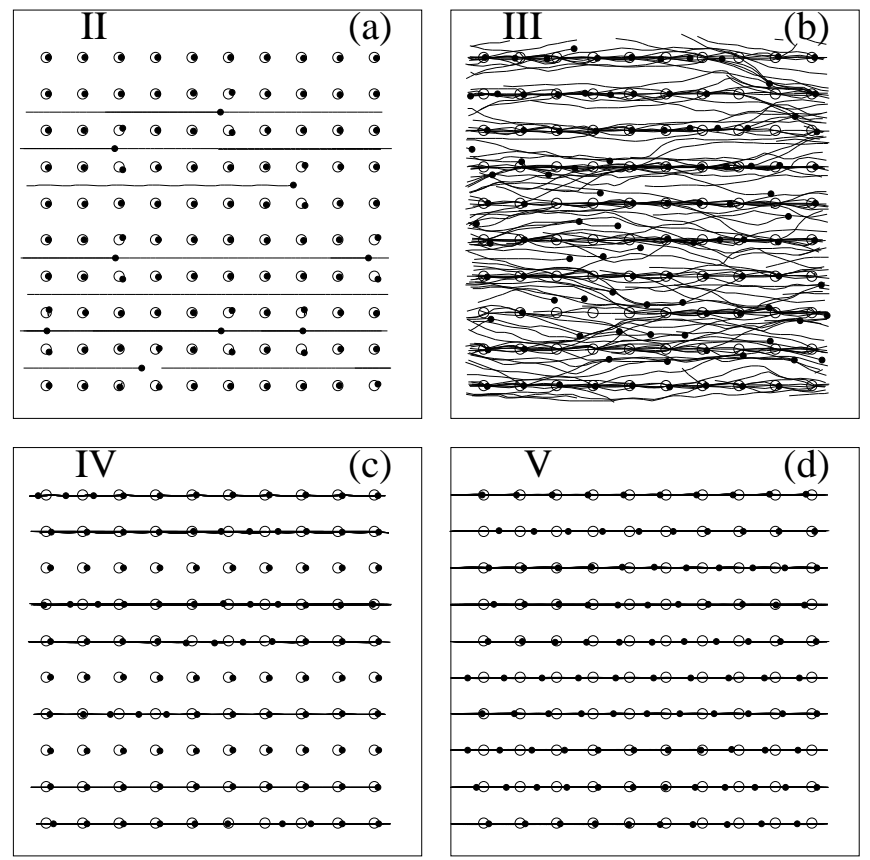

FIG. 2. Trajectories of the east-bound flowing vortices, for the voltage-current curve in Fig. 1, for regions: II, interstitial flow (a); III, disordered flow (b); IV, incommensurate 1D flow (c); and V, moving (in)commensurate rows (d). The vortices are represented by black dots and pinning sites by open circles. For clarity, only a $20 \lambda \times 20 \lambda$ subset of the $36 \lambda \times 36 \lambda$ sample is shown.
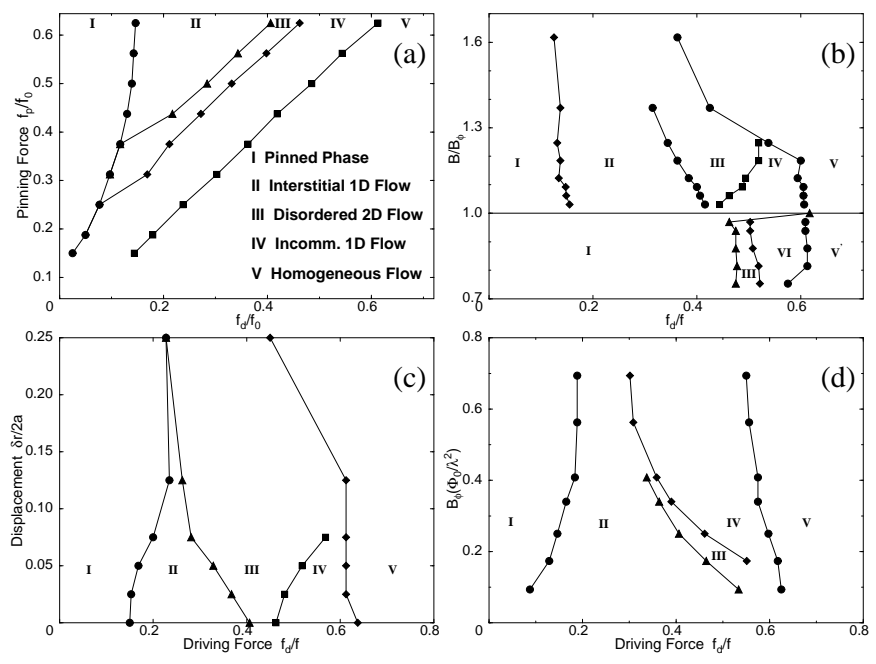

FIG. 3. Dynamic phase diagrams with a square pinning lattice. Here, $B / B_{\phi}=1.062, B_{\phi}=0.25 \Phi_{0} / \lambda^{2}, r_{p}=0.3 \lambda$, and $f_{p}=0.625 f_{0}$, unless otherwise noted. (a) Pinning force $f_{p}$ versus driving force $f_{d}$. As $f_{d}$ is increased, the phase boundaries II-III, III-IV, and IV-V become linear. (b) $B / B_{\phi}$ versus $f_{d}$. For $B / B_{\phi}>1$, regions I through $\mathrm{V}$ can be observed, with the disordered region III growing and the ordered-flow regions II and IV reducing in size. A similar situation occurs in (c) for gradually increasing the amount of disorder in the location of the pins. (d) Dynamic phase diagram for $B_{\phi}$ versus $f_{d}$. 